\title{
Development of a theory-informed implementation intervention to improve the triage, treatment and transfer of stroke patients in emergency departments using the Theoretical Domains Framework (TDF): the $T^{3}$ Trial
}

Louise E. Craig ${ }^{1}$, Natalie Taylor ${ }^{2}$, Rohan Grimley ${ }^{3}$, Dominique A. Cadilhac ${ }^{4,5}$, Elizabeth Mclnnes ${ }^{1}$, Rosemary Phillips ${ }^{1}$, Simeon Dale ${ }^{1}$, Denise O'Connor ${ }^{6}$, Chris Levi ${ }^{7,8}$, Mark Fitzgerald ${ }^{9,10,11}$, Julie Considine ${ }^{12}$, Jeremy M. Grimshaw ${ }^{13,14}$, Richard Gerraty ${ }^{15}$, N. Wah Cheung ${ }^{16}$, Jeanette Ward ${ }^{17,18}$ and Sandy Middleton ${ }^{1 *}$

\begin{abstract}
Background: Theoretical frameworks and models based on behaviour change theories are increasingly used in the development of implementation interventions. Development of an implementation intervention is often based on the available evidence base and practical issues, i.e. feasibility and acceptability. The aim of this study was to describe the development of an implementation intervention for the $T^{3}$ Trial (Triage, Treatment and Transfer of patients with stroke in emergency departments (EDs)) using theory to recommend behaviour change techniques (BCTs) and drawing on the research evidence base and practical issues of feasibility and acceptability.
\end{abstract}

Methods: A stepped method for developing complex interventions based on theory, evidence and practical issues was adapted using the following steps: (1) Who needs to do what, differently? (2) Using a theoretical framework, which barriers and enablers need to be addressed? (3) Which intervention components (behaviour change techniques and mode(s) of delivery) could overcome the modifiable barriers and enhance the enablers? A researcher panel was convened to review the list of $\mathrm{BCTs}$ recommended for use and to identify the most feasible and acceptable techniques to adopt.

Results: Seventy-six barriers were reported by hospital staff who attended the workshops (step 1: thirteen TDF domains likely to influence the implementation of the $T^{3}$ Trial clinical intervention were identified by the researchers; step 2: the researcher panellists then selected one third of the BCTs recommended for use as appropriate for the clinical context of the ED and, using the enabler workshop data, devised enabling strategies for each of the selected BCTs; and step 3: the final implementation intervention consisted of $27 \mathrm{BCTs}$ ).

(Continued on next page)

\footnotetext{
* Correspondence: Sandy.Middleton@acu.edu.au

${ }^{1}$ Nursing Research Institute, St Vincent's Health Australia (Sydney) and

Australian Catholic University, Executive Suite, Level 5 deLacy Building, St

Vincent's Hospital, 390 Victoria Street, Darlinghurst 2010, New South Wales,

Australia

Full list of author information is available at the end of the article
} 
(Continued from previous page)

Conclusions: The TDF was successfully applied in all steps of developing an implementation intervention for the $\mathrm{T}^{3}$ Trial clinical intervention. The use of researcher panel opinion was an essential part of the BCT selection process to incorporate both research evidence and expert judgment. It is recommended that this stepped approach (theory, evidence and practical issues of feasibility and acceptability) is used to develop highly reportable implementation interventions. The classifying of BCTs using recognised implementation intervention components will facilitate generalisability and sharing across different conditions and clinical settings.

Keywords: Implementation intervention, Theoretical Domains Framework, Behaviour change techniques

\section{Background}

Evidence-based guideline recommendations are available for the early management of patients with acute stroke. Early diagnosis of stroke in emergency departments (EDs); administration of recombinant tissue plasminogen activator (rt-PA) and endovascular clot retrieval to eligible patients; and management of fever, hyperglycaemia and swallowing difficulties before transfer to a stroke unit are essential elements of evidence-based stroke care and recommended in current clinical guidelines [1]. Yet, inappropriate triage [2] and delays in diagnosis, treatment and transfer of stroke patients from ED to stroke units still occur [1]. The $\mathrm{T}^{3}$ Trial is a prospective, multicentre, parallel group, blinded, cluster randomised trial that aimed to evaluate the effectiveness of an implementation intervention to improve the triage, treatment and transfer of stroke patients from ED to stroke units on 90-day outcomes and in-hospital processes of care [3]. This paper describes the development of the theorybased implementation intervention for this Trial.

The use of theory in the intervention development process has been identified by the UK Medical Research Council (MRC) as crucial to increase intervention effectiveness by targeting causal determinants of behaviour and facilitate an understanding of what works (i.e. the mechanisms of change) [4, 5]. Several approaches have been proposed that integrate the use of theory in implementation intervention development [5-7]. Although there are studies that apply these approaches in the process of developing an implementation intervention [8-10], frequently, these interventions are still based on intuitive or non-theoretical methods [11]. There is also a lack of detailed reporting of the process of intervention development and the content of the implementation intervention which, if available, would assist replication and advance the knowledge base about the optimum approach for intervention development [12].

The Theoretical Domains Framework (TDF) is a framework of 14 theoretical domains derived from 33 behaviour change theories developed using a process of expert consensus with subsequent validation work $[13,14]$. The TDF has successfully been applied in a number of healthcare settings to (i) guide intervention development for the implementation of guidelines or clinical interventions $[6,9,15]$, (ii) characterise, according to theory, an existing intervention to implement evidence-based care to facilitate accurate replication [16, 17], and (iii) understand factors that may inhibit uptake of an intervention $[18,19]$. The additional benefit of the TDF is that behaviour change techniques (BCTs) have been preassigned to each of the TDF domains [20]. Two matrices which assign the most appropriate BCTs to each of the TDF domains have previously been developed by Cane et al. [20] and by Michie et al. [5].

The implementation of complex clinical interventions, such as those that have numerous intervention components, as is the case in the $\mathrm{T}^{3}$ Trial, often involve the use of theory but may also require incorporation of the evidence base and consideration of practical issues such as feasibility and acceptability [21]. Firstly, theory is important to understand the factors influencing clinician behaviours and to guide the use of appropriate behavioural change techniques $(\mathrm{BCT})$, the smallest components of an implementation intervention [6]. Secondly, evidence regarding technique effectiveness can assist the selection of BCTs and the best mode of delivery [6]. This might be generic behaviour change evidence but also might incorporate context-specific evidence, from the stroke or ED literature in the case of this study. Thirdly, an understanding of practical issues (feasibility and acceptability) and expert clinical judgment can guide the selection of the most relevant BCTs for a particular context [6]. Some studies have incorporated stakeholder opinion in the design of implementation interventions to incorporate practical considerations and judgment $[6,9,10,22]$. As contextual issues have a significant influence on the delivery and impact of complex clinical interventions [4], a theory-based evidence-driven approach which takes into account context should be considered in developing implementation interventions. However, there are very few well reported studies that use this stepped approach of intervention development (theory, evidence and practical issues of feasibility and acceptability).

The aim of this study was to describe the development of an implementation intervention (i) using theory to inform selection of BCTs for the $\mathrm{T}^{3}$ Trial, (ii) further 
guided by the evidence of effectiveness of implementation interventions (including that from a previous acute stroke implementation trial [23]) and (iii) consideration of researcher opinion to select appropriate BCTs. This implementation intervention will be subsequently tested under trial conditions [3].

\section{Methods}

The $\mathrm{T}^{3}$ Trial clinical intervention is an evidence-based care bundle of clinical protocols for triage, treatment and transfer of patients following acute stroke and comprised of 12 different clinical care elements (hereonin referred to as 'target behaviours' [Table 1]) [3]. As the $\mathrm{T}^{3}$ trial clinical intervention consisted of 'a number of separate elements which seem essential to the proper functioning of the intervention although the 'active ingredient' of the intervention that is effective is difficult to specify', it meets the MRC definition of a complex intervention [4]. Four of the five-stepped method proposed by French et al. [6] for developing complex interventions based on theory, evidence and practical issues were undertaken as follows:

- Step 1: Who needs to do what, differently?

- Step 2: Using a theoretical framework, which barriers and enablers need to be addressed?

- Step 3: Which intervention components (behaviour change techniques and mode(s) of delivery) could overcome the modifiable barriers and enhance the enablers?
Step 4: 'How can behaviour change be measured and understood?' previously has been reported in our published protocol paper (primary and secondary outcomes with an a priori planned process evaluation) [3]. Step 5: 'How can behaviour change be sustained' is beyond the scope of the $\mathrm{T}^{3}$ Trial.

\section{Step 1: Who needs to do what, differently?}

Twelve evidence-based targeted behaviours were identified by the trial investigators for the triage, treatment and transfer $\left(\mathrm{T}^{3}\right)$ elements of the intervention. We selected the target clinical behaviours to be addressed, based on documented evidence-practice gaps. As per French et al.'s approach we specified the target behaviours in detail by asking the following questions: What is the clinical behaviour that you will try to change? Who performs the behaviour(s)? And when and where do they perform the behaviour(s)?

\section{Step 2: Using a theoretical framework, which barriers and enablers need to be addressed?}

One barrier and enabler multidisciplinary workshop (1-h duration) was conducted at each of the thirteen $\mathrm{T}^{3}$ Trial intervention hospitals across three Australian states and the Australian Capital Territory between October 2014 and December 2014. Purposive sampling was used to select workshop participants who could provide detailed feedback on barriers and enablers to the $\mathrm{T}^{3}$ Trial clinical intervention, namely (i) senior healthcare professionals working in ED (e.g. emergency physician, emergency

Table 1 Target clinical behaviours for $\mathrm{T}^{3}$ trial

\begin{tabular}{|c|c|c|}
\hline Target behaviour & $\begin{array}{l}\text { Target clinical behaviour (includes timepoint if not immediate) } \\
\text { Location: emergency department }\end{array}$ & Who performs the behaviour \\
\hline Triage & $\begin{array}{l}\text { All patients presenting to ED with signs and symptoms } \\
\text { of suspected acute stroke should be triaged as Australian } \\
\text { Triage Scale Category } 1 \text { or } 2 \text { (seen within } 10 \text { min) }\end{array}$ & ED nurse \\
\hline Thrombolysis & $\begin{array}{l}\text { All patients to be assessed for rt-PA eligibility in ED } \\
\text { All eligible patients to receive rt-PA in ED }\end{array}$ & $\begin{array}{l}\text { ED nurse, ED doctor, Stroke doctor, Stroke nurse } \\
\text { ED doctor, Stroke doctor, Stroke nurse }\end{array}$ \\
\hline $\begin{array}{l}\text { Temperature } \\
\text { management }\end{array}$ & $\begin{array}{l}\text { All patients to have their temperature taken on admission } \\
\text { to ED and then at least } 4 \text { hourly whilst they remain in ED } \\
\text { Temperature } 37.5^{\circ} \mathrm{C} \text { or greater to be treated with paracetamol } \\
\text { (acetaminophen) in ED }\end{array}$ & $\begin{array}{l}\text { ED nurse } \\
\text { ED nurse }\end{array}$ \\
\hline $\begin{array}{l}\text { Blood glucose } \\
\text { management }\end{array}$ & $\begin{array}{l}\text { Venous BGL sample taken to laboratory on admission to ED } \\
\text { Finger prick BGL recorded on admission to ED and finger } \\
\text { prick BGL monitored every } 6 \mathrm{~h} \text { (or greater if elevated) } \\
\text { Insulin administered to all patients with } B G L>10 \mathrm{mMol} / \mathrm{L} \\
\text { within } 1 \mathrm{~h} \text { in ED or stroke unit }\end{array}$ & $\begin{array}{l}\text { ED nurse, ED doctor } \\
\text { ED nurse, Stroke nurse } \\
\text { ED nurse, Stroke nurse, Endocrinologist }\end{array}$ \\
\hline Swallow management & $\begin{array}{l}\text { Patients to remain NBM until a swallow screen by non-Speech } \\
\text { pathologist or swallow assessment by Speech pathologist } \\
\text { performed in ED } \\
\text { All patients who fail the swallow screen to remain NBM } \\
\text { and have a swallowing assessment by a Speech pathologist } \\
\text { whilst in ED }\end{array}$ & $\begin{array}{l}\text { ED nurse, Stroke nurse, ED doctor, Speech pathologist } \\
\text { Speech pathologist }\end{array}$ \\
\hline Transfer & $\begin{array}{l}\text { All patients with stroke to be discharged from ED within } 4 \mathrm{~h} \\
\text { All patients with stroke to be admitted to the hospital's stroke unit }\end{array}$ & $\begin{array}{l}\text { ED nurse, ED doctor, Stroke nurse, Bed manager } \\
\text { ED nurse, Stroke nurse, Bed manager }\end{array}$ \\
\hline
\end{tabular}


nurses) or in stroke units (e.g. stroke physician, stroke nurses, endocrinologists, speech pathologists and bed managers) and (ii) involved in routine delivery of the target behaviours. The workshops were aimed at identifying the perceived barriers and enablers that may influence the uptake of each of the target behaviours. The workshops were facilitated by SD and SM with assistance from emergency, neurology and endocrine physician $\mathrm{T}^{3}$ Trial researchers. A standard presentation was given at the workshops to provide consistent information about each of the target behaviours. The workshop participants were asked to nominate specific barriers for each of the behaviours and specific enablers and strategies that could be used to overcome the barriers.

The workshops were audio recorded and transcribed verbatim. The interview transcripts were coded using thematic analysis by a single coder (LC) according to the TDF domains [14]. Individual barriers were classified to the relevant domain of the TDF. The constructs, that is the concepts provided for each of the TDF domains, were used to assist interpretation and to ensure accurate assignment of the TDF domains. The coding framework was devised by the lead author (LC) and RP applied this framework to a subset of transcripts $(n=5)$ to test the interpretation of the codes. A third researcher (NT), with expertise in the application of the TDF to primary data, independently checked the assignment of all transcript data to the TDF domains. It was agreed that should a number of barriers be reflected by more than one TDF domain, the most relevant domain should be selected. Discrepancies in allocation were resolved by reviewing and discussing the transcript dialogue before and after the extract. The TDF constructs and contextual information reported for an individual barrier/enabler were also used to understand and resolve any discrepancies.

\section{Step 3: Which intervention components (behaviour change techniques and mode(s) of delivery) could overcome the modifiable barriers and enhance the enablers?}

The Cane et al. matrix [20] which recommended the most appropriate BCTs to each of the TDF domains was primarily used to identify BCTs. Two domains (memory, attention and decision processes and social/professional role and identity) do not have any specified BCTs in the Cane et al. matrix; therefore, a similar matrix previously developed by Michie et al. was used to inform the BCTs for these domains and other domains where the recommended BCTs were considered more appropriate than that recommended by the Cane et al. matrix. The BCT taxonomy [24] was also provided to the researchers as a resource to use where neither of the two matrices identified an appropriate BCT. Whilst these tools are useful for assigning relevant $\mathrm{BCTs}$, they do not incorporate the evidence regarding implementation intervention effectiveness or issues of feasibility or acceptability. To address this, we used a pragmatic approach to selecting BCTs using the $\mathrm{T}^{3}$ Trial investigators knowledge of the clinical intervention and experience of the clinical context. A panel of five $\mathrm{T}^{3}$ Trial investigators and researchers (SM, DC, RG [a stroke physician], RP and ES), all of which had experience of applying the TDF in stroke implementation research, independently reviewed the list of matrixassigned BCTs and from this identified the most feasible and acceptable techniques for the $\mathrm{T}^{3}$ Trial.

It was identified that the selection of $\mathrm{BCTs}$ for a single behaviour would be time consuming likely taking up to two and half hours to complete; therefore, due to time constraints of the researcher panellists, it was considered unfeasible for them to complete the selection process for all 12 behaviours. Instead, the panel were instructed to select BCTs to address the barriers associated with only one of the target behaviours Administration of insulin to all patients with $B G L>10 \mathrm{mMol} / \mathrm{L}$ within 1 hour by insulin infusion. This behaviour was selected as it represented nearly every TDF domain (11 out of 13) mapped in step 1 which would allow the findings to then be applied to the remaining behaviours. The panel were provided with a number of resources (shown in brackets below) and specifically asked to

1. Identify the BCTs considered appropriate (feasible and acceptable to clinicians) e.g. techniques that are time efficient in an ED setting; resource 1: barrier extracts and TDF definitions [Additional file 1]; resource 2: domains with corresponding $\mathrm{BCT}$ and definitions [Additional file 2]; resource 3: technique definition and examples [Additional file 3]; resource 4: enabler data from the barrier and enabler workshops [Additional file 4])

2. Identify the BCTs considered inappropriate (not feasible and unacceptable to clinicians) e.g. techniques that reduce the need for clinical decision-making; resources 1 to 4

3. Identify any TDF domains where none of the BCTs identified by the primary Cane et al. matrix [20] were viewed as appropriate; resources 2 and 3

4. Identify further BCTs not selected by the primary Cane et al. matrix [20] e.g. techniques identified in other BCT matrices and taxonomies $[5,24]$ that were considered to be more appropriate; resources 2 and 3

5. Devise strategies to operationalise the selected BCTs; resource 5: table of evidence [9] to present different modes of delivery, e.g. face-to-face education meetings and local opinion leaders, based on Cochrane Effective 
Practice and Organisation of Care [EPOC] reviews (Additional file 5) [25-30]

In summary, each of the researchers were asked to select the most appropriate $\mathrm{BCT}$ based on the following criteria; feasibility of use in the ED context, personal experience of use, local relevance and acceptability. An evidence table providing effectiveness data for commonly used modes of delivery such as face-to-face education meetings and local opinion leaders was included to assist the researchers in suggesting strategies to operationalize the BCTs in an ED context. Relevant qualitative data extracts generated from the barrier and facilitator workshops were also included to allow the researchers to assess the feasibility of using the technique in ED (further details can be provided on request). An overview of this process is provided in Fig. 1. As the TDF domains mapped in step 2 were also represented in the remaining 11 behaviours, the researchers were advised that their selections would inform the final set of BCTs to be applied across all the behaviours. The researcher completed the task independently with the lead author (LC) available to guide the researchers through the process and respond to any questions to ensure adherence to the instructions provided. A BCT was included in the final set if it was selected by at least one of the researchers. The BCTs were tabulated and the frequency of selection by the researchers was reported. The selections of the researcher panel were applied by LC to the remaining 11 behaviours to create a final set of BCTs for the $\mathrm{T}^{3}$ Trial implementation intervention. In order to increase the transferability of the reporting of the implementation intervention, the final set of BCTs were classified by LC according to general evidence-based intervention components (BCTs and mode of delivery) commonly reported in the implementation literature [23]. These were as follows: multidisciplinary barrier and enabler workshop [31], interactive and didactic education programme [32, 33], use of opinion leaders [25], reminders [34] and site support [34]. Key relevant literature examples which presented BCTs by intervention components were used to classify accordingly $[6,9]$.

\section{Results}

Step 1: Who needs to do what, differently?

The target behaviours for the $\mathrm{T}^{3}$ trial intervention arose from an extensive literature review and the stroke guidelines. These targeted behaviours along with who performs the behaviour, the timepoint and the location that the behaviour occurs are listed in Table 1 . These target behaviours were chosen because they had supporting evidence and were potentially modifiable at a clinician level.

\section{Step 2:}

Thirteen workshops were conducted with 105 staff from 13 hospitals. Workshop group size ranged from minimum of five participants to maximum of 11 participants. These multidisciplinary workshops were facilitated by the researchers and included senior nurses from ED and stroke units, medical practitioners (ED physicians, neurologists, endocrinologists and their junior doctors),

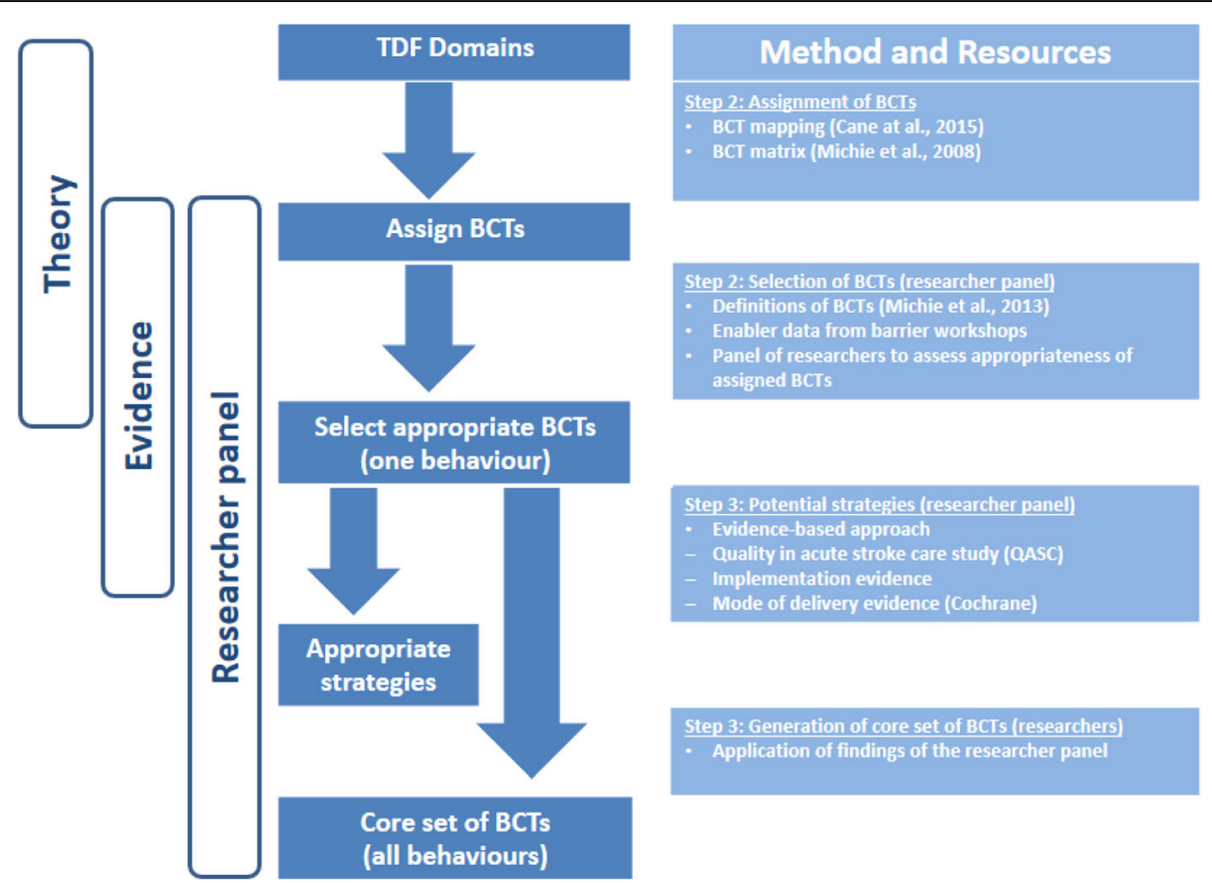

Fig. 1 Selecting behavioural change techniques and strategies to inform the $T^{3}$ Trial implementation intervention 
speech pathologists, and nurse managers. Seventy-six barriers were identified by participants (Table 2). All barriers were mapped to at least one TDF domain. For example, barriers relating to the knowledge domain were associated with gaps in staff's understanding, awareness or content knowledge for certain target behaviours. This resulted in clinical uncertainty and the conduct of practices not compliant with national guidelines. Barriers relevant to the skills domain were related to the conduct of a specific task such as a swallow screen or a lack of skill development opportunity. Barriers assigned to the social/professional role and identity domain were related to professional boundaries, i.e. limited prescribing rights for nurses and professional identity, i.e. opposition to blurring of roles. Barriers relevant to the beliefs about capabilities domain were related to professional confidence, i.e. decision-making or low self-esteem to perform tasks related to the target behaviour. The main area of disagreement between the researchers conducting the mapping related to overlap between two domains, beliefs about capabilities and social/professional role and identity. For example, one researcher understood a barrier to conducting a clinical task to be related to a clinician's self-confidence so mapped the extract to beliefs about capabilities domain whilst the other researcher understood the barrier to be related to professional boundaries, i.e. the task was not traditionally undertaken by that professional group so mapped the extract to social/professional role and identity. Areas of disagreement were resolved by discussion between the two researchers. No barriers were assigned to the intentions domain. Furthermore, the same barrier was often reported for more than one of the behaviours, for example, competing priorities in a busy emergency department environment was reported for four different behaviours. Overall, the same nine barriers were reported for the different target behaviours.

\section{Step 3: Which intervention components (behaviour change techniques and mode(s) of delivery) could overcome the modifiable barriers and enhance the enablers?}

The panel selected appropriate BCTs for the 11 barriers identified for the target behaviour provided (Table 3). There was no TDF domain where the assigned BCTs based on the Cane et al. matrix [20] were viewed as inappropriate by the researchers. Overall, 22 of the selected BCTs for all 11 barriers were based on the Cane et al. matrix [20], with the remaining five selected BCTs based on the Michie et al. matrix [5].

\section{Generating a final set of BCTs}

The findings from the researcher panel were then applied to the remaining 11 behaviours to generate a final set of BCTs to apply to all the behaviours. Two TDF domains (beliefs about capabilities and reinforcement) were not represented by any of the 11 barriers used in the researcher panel; therefore, selection of the most appropriate BCTs was based on the triallists experience [6]. The final set of BCTs $(n=27)$ are reported by general implementation intervention components in Table 4. Some techniques, e.g. action planning and coping skills, were classified into more than one relevant implementation intervention component.

\section{Discussion}

The barriers that hospital staff believed to be likely to influence the implementation of the $\mathrm{T}^{3}$ Trial clinical protocol were mapped to 13 TDF domains. These domains were used to guide the content of an implementation intervention consisting of $27 \mathrm{BCTs}$. It is anticipated that by using this systematic, theory-based approach to inform the content of an implementation intervention the potential for effectiveness in changing behaviour will be optimised. Researcher opinion, together with the existing research evidence base, was used to refine the theoretically driven intervention framework by selecting appropriate BCTs and suggesting strategies to operationalise the BCTs in an ED context. One of the few studies that used the TDF and BCTs to define the content of an intervention to change patient's compliance in bronchiectasis used an adapted scoring system from Michie et al. to select BCTs from a longer list [22]. Experts classified BCTs into one of the following categories which then generated the scores: agreed use, agreed non-use, disagreement and uncertain. The validity of using such criteria in the BCT selection process was not reported by the authors, indicating that further research is required in this area. The use of stakeholder opinion has been used in another study to define how the proposed intervention could be delivered as opposed to selecting the BCTs [22]. This process of incorporating the views of researchers with experience of the clinical context and knowledge of the clinical intervention is likely to enhance the clinical acceptability of the implementation intervention. The BCTs selected by the researchers aligned with the BCTs recommended by the matrix, suggesting that this is a valuable tool to use to highlight relevant BCTs.

\section{Limitations}

The final set of BCTs was generated using the researcher panellists' selections based on one target behaviour. This assumes that the BCTs considered appropriate for one behaviour have applicability to target similar barriers associated with the other behaviours. Potentially, appropriate BCTs to target different barriers for the other behaviours may not have been included. However, the 


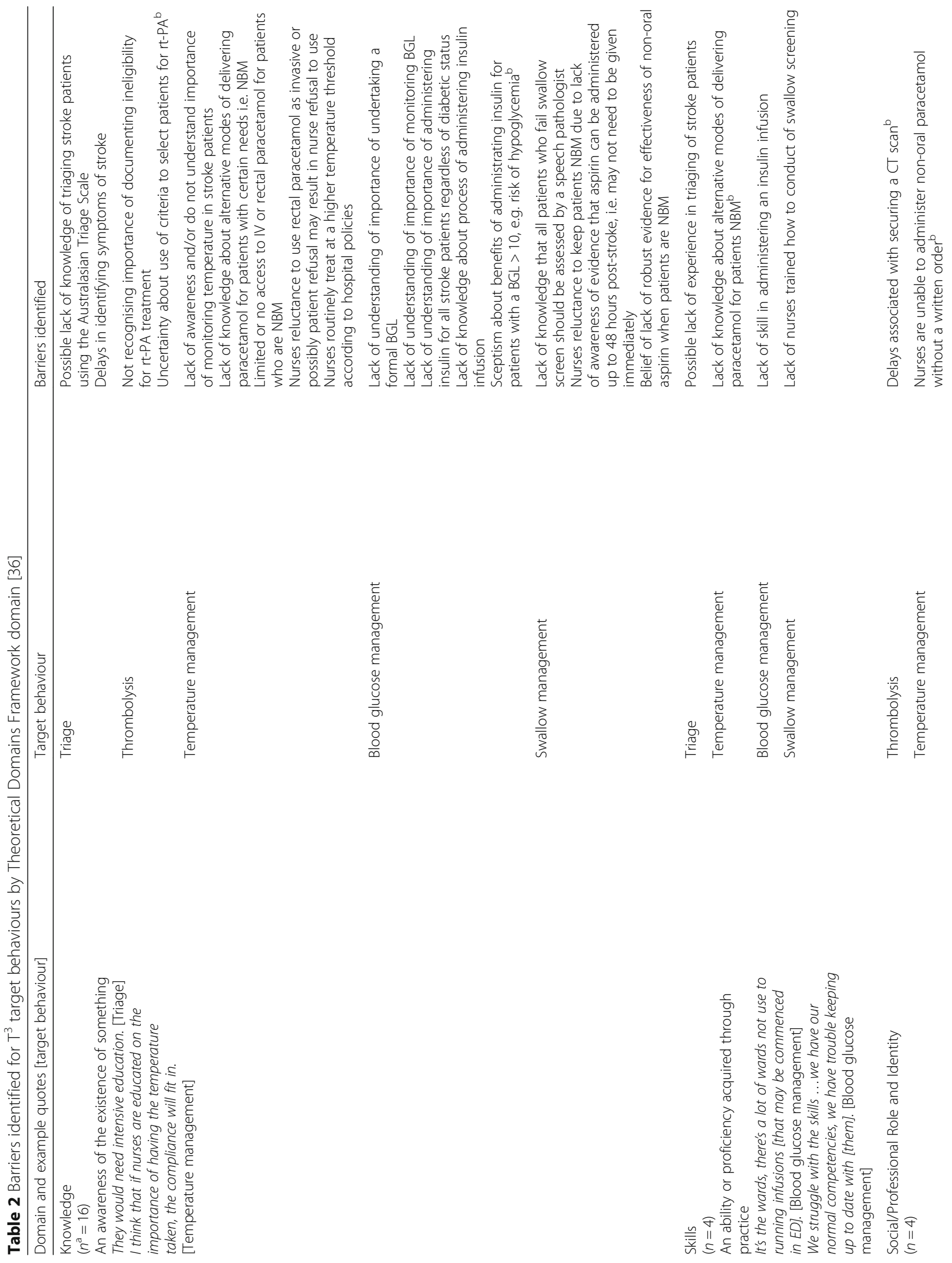




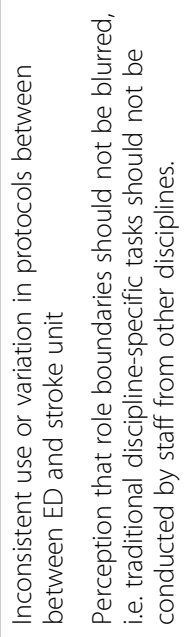
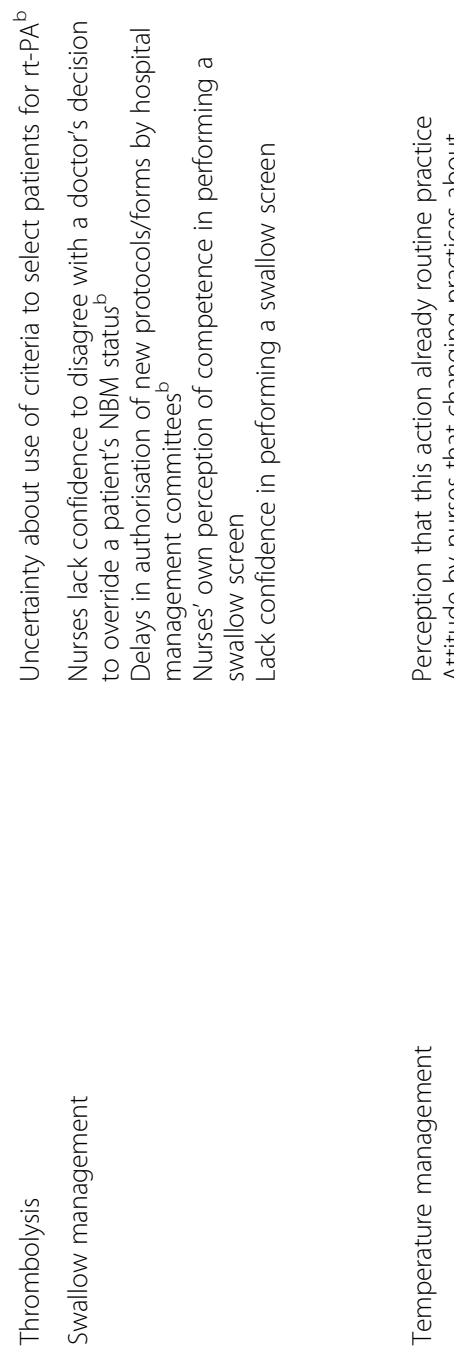
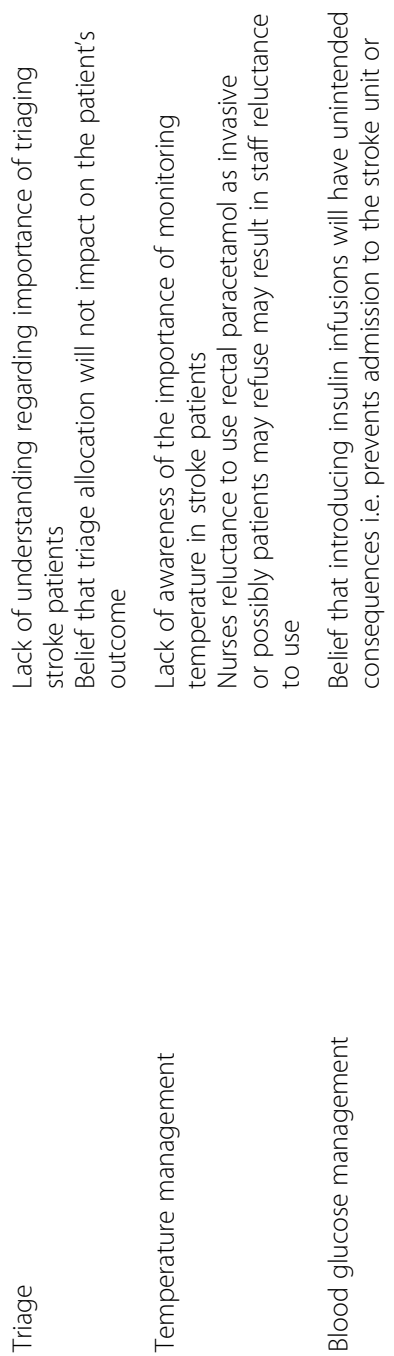

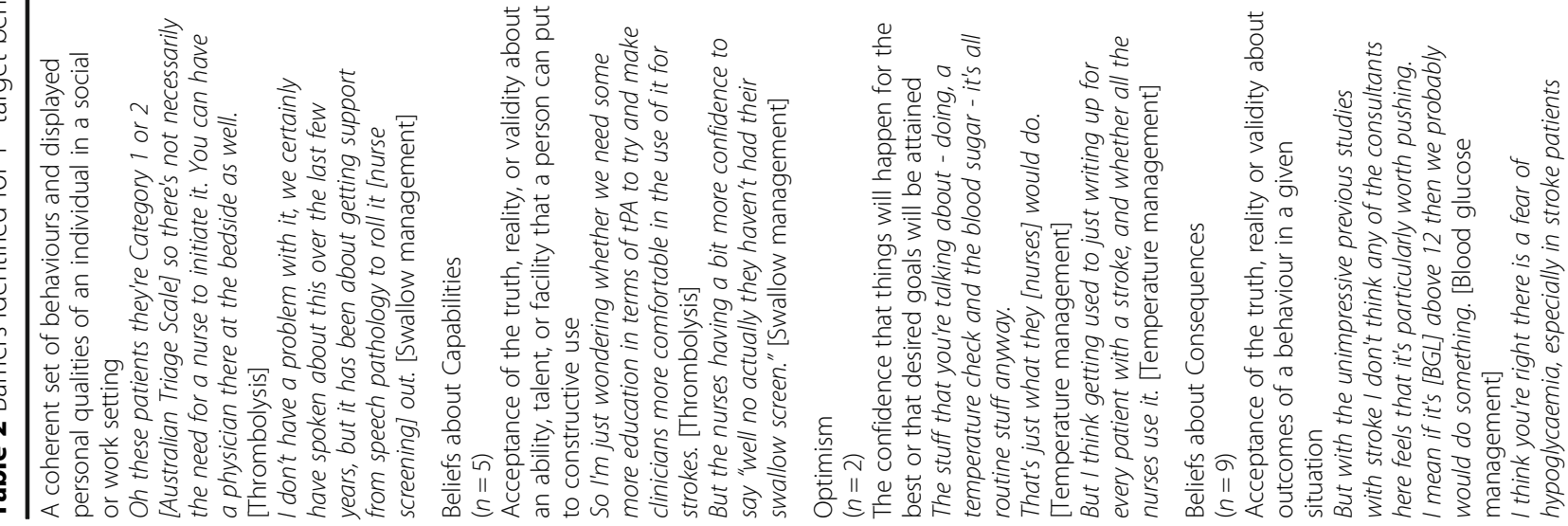



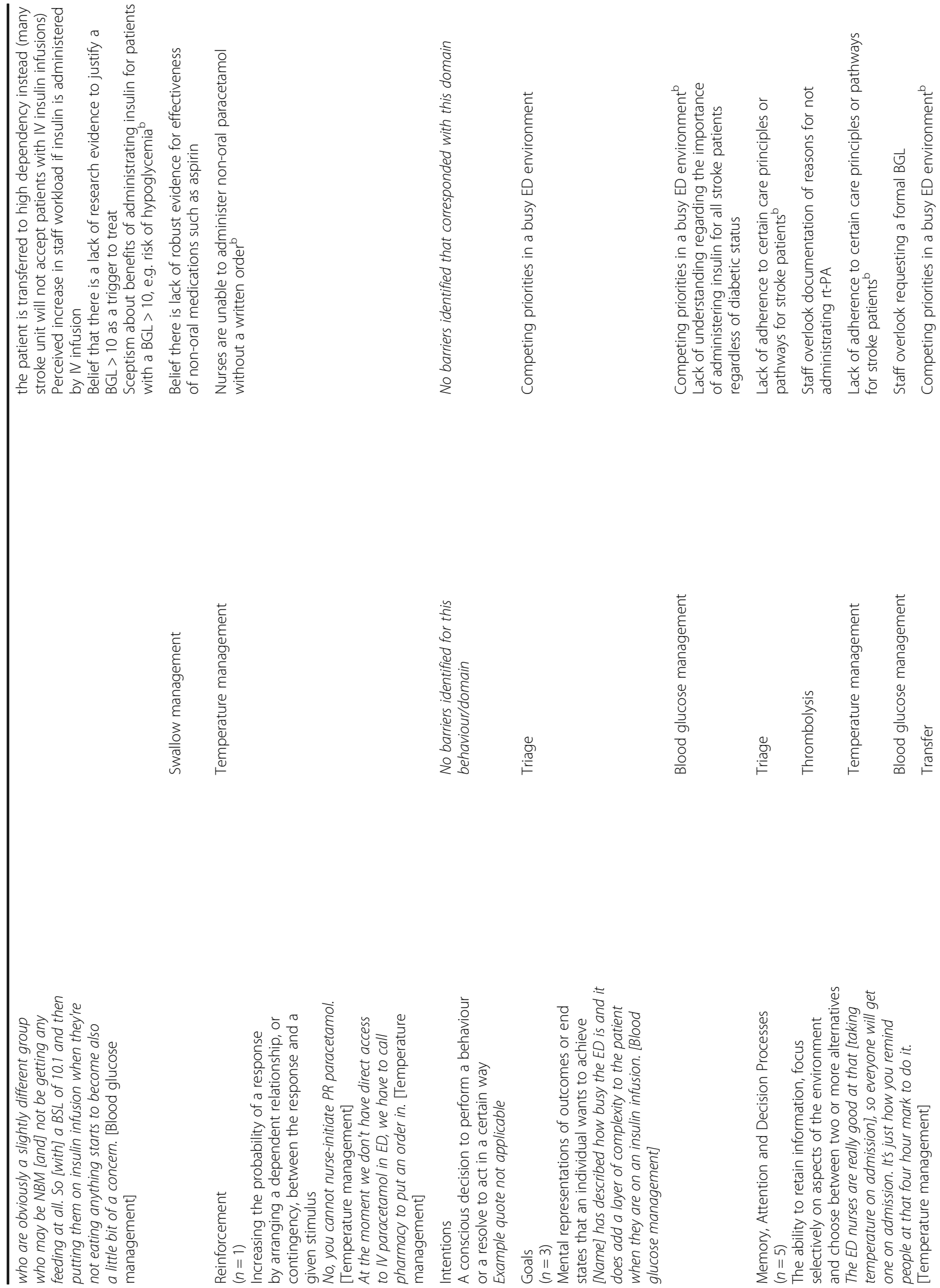

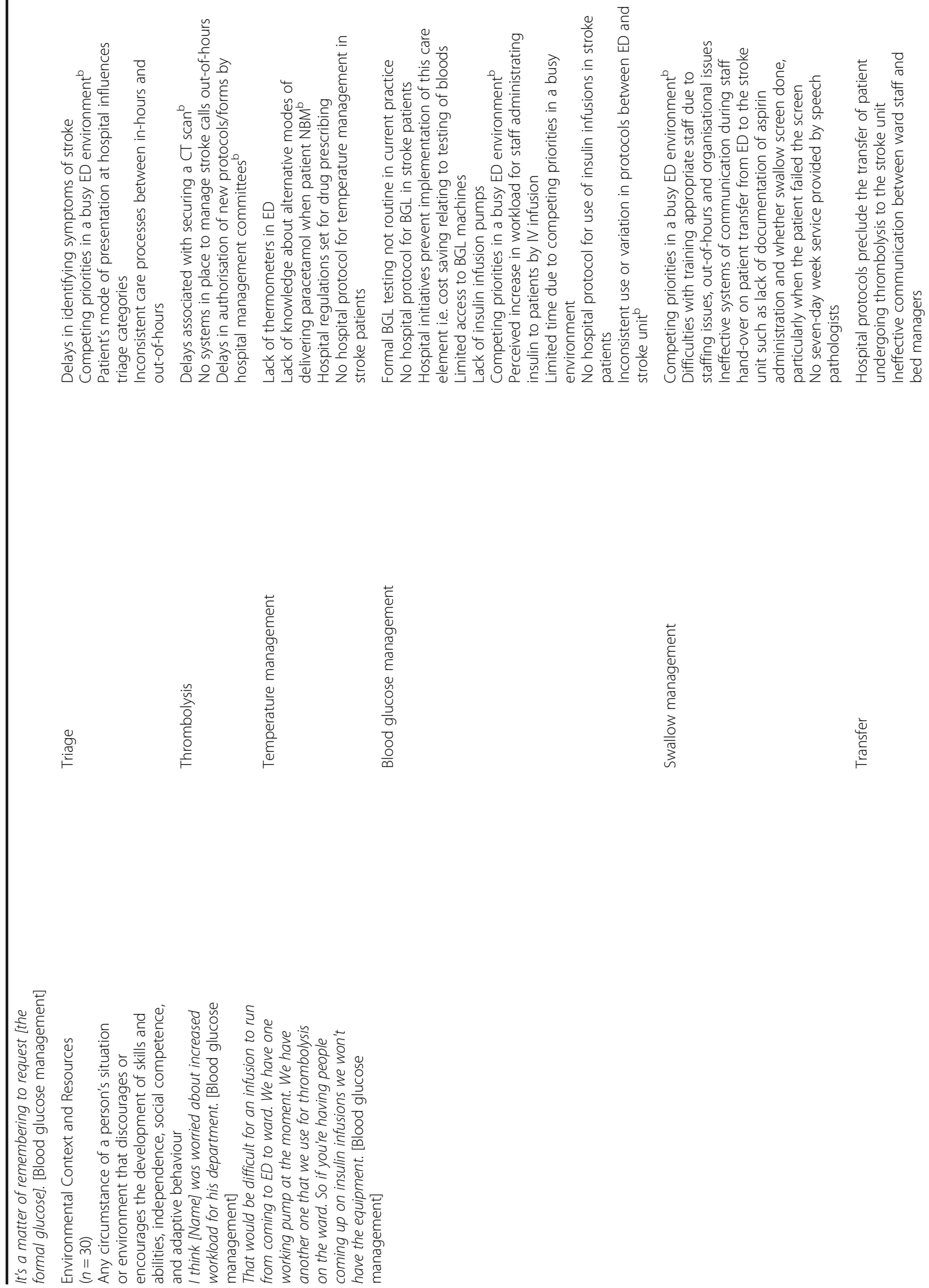
Craig et al. Implementation Science (2017) 12:88

Page 11 of 17

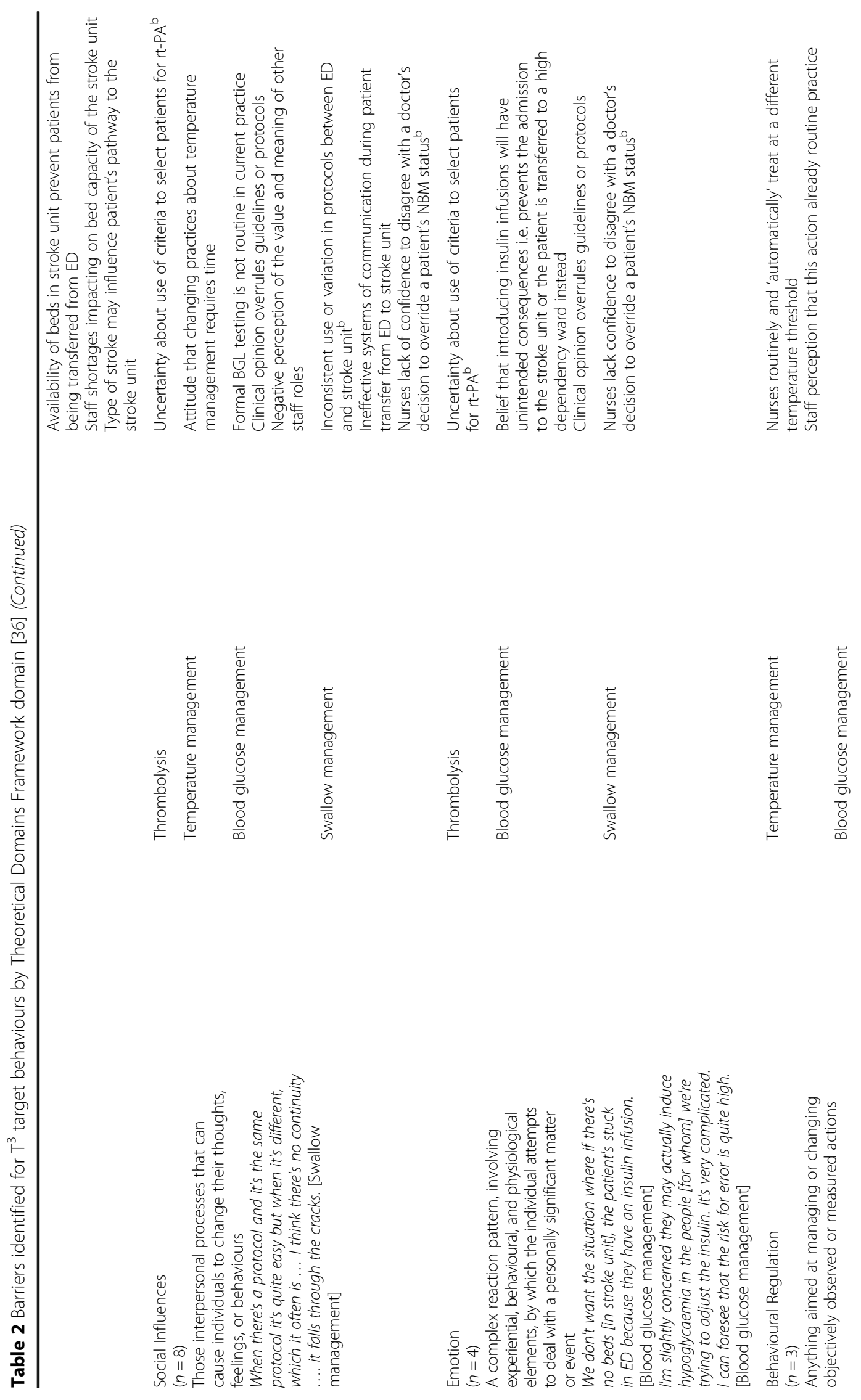




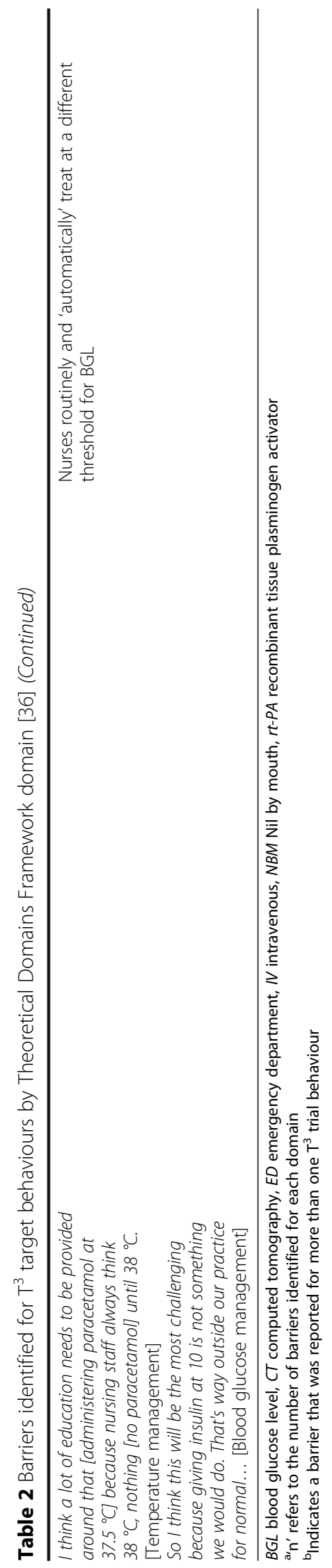


Table 3 Behaviour change techniques mapped to the Theoretical Domain Framework identified for intravenous insulin infusion barriers

\begin{tabular}{ll}
\hline Domain & Corresponding techniques $^{\mathrm{a}}$ \\
\hline Knowledge & Health consequences \\
& Feedback on behaviour \\
& Behavioural rehearsal/practice \\
Goal/target specified: behaviour or outcome \\
Self-monitoring
\end{tabular}

Social/professional role and identity
Social support or encouragement

Salience of consequences

Anticipated regret

Social and environmental consequences

Comparative imagining of future outcome

Pros and cons

Persuasive communication

Feedback on behaviour

Goal setting (behaviour)

Action planning (including implementation intentions)

Memory, Attention and Decision Planning, implementation Processes

Prompts, triggers, cues

Environmental context and resources

Restructuring the social environment

Prompts/cues

Avoidance/changing exposure to cues for the behaviour

Environmental changes (e.g. objects to facilitate behaviour)
Definition of technique

Provide information (e.g. written, verbal, visual) about health consequences of performing the behaviour

Monitor and provide informative or evaluative feedback on performance of the behaviour (e.g. form, frequency, duration, intensity)

Prompt practice or rehearsal of the performance of the behaviour one or more times in a context or at a time when the performance may not be necessary, in order to increase habit and skill

Set a goal defined in terms of the behaviour to be achieved

Establish method for the person to monitor and record their behaviour(s) as part of behaviour change strategy

Advise on, arrange or provide social support (e.g. from friends, relatives, colleagues, 'buddies' or staff) or non-contingent praise or reward for performance of the behaviour. It includes encouragement and counselling, but only when it is directed at the behaviour

Use methods specifically designed to emphasise the consequences of performing the behaviour with the aim of making them more memorable (goes beyond informing about consequences)

Induce or raise awareness of expectations of future regret about performance of the unwanted behaviour

Provide information (e.g. written, verbal, visual) about social and environmental consequences of performing the behaviour

Prompt or advise the imagining and comparing of future outcomes of changed versus unchanged behaviour

Advise person to identify and compare reasons for wanting (pros) and not wanting (cons) to change behaviour

Credible source presents arguments in favour of the behaviour

Monitor and provide informative or evaluative feedback on performance of the behaviour (e.g. form, frequency, duration, intensity)

Set a goal defined in terms of the behaviour to be achieved

Prompt detailed planning of performance of behaviour (must include $\geq$ one of context, frequency, duration and intensity). Context may be environmental (physical or social) or internal (physical, emotional or cognitive)

Prompt detailed planning of the behaviour goal (including at least one of context, frequency, intensity and duration of performance)

Use environmental, social or internal stimuli to prompt or cue performance of wanted behaviour or non-performance of unwanted behaviour

Change, or advise to change the social environment in order to facilitate performance of the wanted behaviour or create barriers to the unwanted behaviour (other than prompts/cues, rewards and punishments)

Introduce or define environmental or social stimulus with the purpose of prompting or cueing the behaviour. The prompt or cue would normally occur at the time or place of performance

Advise on how to avoid exposure to specific social and contextual/ physical cues for the behaviour, including changing daily or weekly routines

Change the environment in order to facilitate the target behaviour (other than prompts, rewards and punishments, e.g. choice of food provided) 
Table 3 Behaviour change techniques mapped to the Theoretical Domain Framework identified for intravenous insulin infusion barriers (Continued)

\begin{tabular}{|c|c|c|}
\hline & & $\begin{array}{l}\text { Explicitly draw attention to others' performance to elicit } \\
\text { comparisons }\end{array}$ \\
\hline & Social support or encouragement (general) & $\begin{array}{l}\text { Advise on, facilitate or provide development of general social } \\
\text { support for the behaviour (e.g. friends, relatives, colleagues, } \\
\text { 'buddies' or staff) }\end{array}$ \\
\hline & Information about others approval & $\begin{array}{l}\text { Provide information about what other people think about the } \\
\text { behaviour. } \\
\text { Clarifies whether others will like, approve or disapprove of what } \\
\text { the person is doing or will do }\end{array}$ \\
\hline & Social support (emotional) & $\begin{array}{l}\text { Advise on or facilitate development of emotional social support } \\
\text { for performing the behaviour }\end{array}$ \\
\hline & Social support (practical) & $\begin{array}{l}\text { Advise on or facilitate development of practical help for } \\
\text { achieving the behaviour }\end{array}$ \\
\hline & Modelling or demonstrating the behaviour & Provide an example for people to aspire to or imitate \\
\hline \multirow[t]{2}{*}{ Emotion } & Reduce negative emotions & $\begin{array}{l}\text { Advise on ways of reducing negative emotions to facilitate } \\
\text { performance of the behaviour }\end{array}$ \\
\hline & Coping skills & $\begin{array}{l}\text { Analyse problem and generate or select solutions that include } \\
\text { overcoming barriers and increasing facilitators }\end{array}$ \\
\hline Behavioural Regulation & Self-monitoring of behaviour & $\begin{array}{l}\text { Establish method for person to monitor and record their } \\
\text { behaviour(s) as part of a behaviour change strategy }\end{array}$ \\
\hline
\end{tabular}

ababel as per matrix by Cane et al. [20]

purpose of the researcher panel was to develop a final set of BCTs that could be used for each of the behaviours. It was considered resource-intensive to apply this selection process for all 12 behaviours in this complex intervention. Acknowledging this limitation, this approach should be considered valid and reliable as a theoretical framework was used to develop the implementation intervention and nearly all the relevant TDF domains (11 out of 13) common to the 12 behaviours were represented by the behaviour used as an example in the researcher panel. The considerable time and resources required in the intervention development process have been raised elsewhere [6] and have implications for applying for research funding, whereby funding bodies often expect the intervention to be fully developed on application, which is usually not possible without separate pre-trial funding, usually difficult to secure.

\section{Strengths}

The barrier and enabler workshops allowed comprehensive identification of relevant barriers and enablers by hospital staff, providing data for each of the $\mathrm{T}^{3}$ Trial behaviours. This ensured that all the individual elements of the $\mathrm{T}^{3}$ Trial clinical intervention were addressed, a necessity for complex interventions. Using the TDF framework enabled the classification of barriers to BCTs required for each $\mathrm{T}^{3}$ Trial behaviour. The TDF is readily being used by researchers to explore behaviour change but few describe the process of how to use theoretical frameworks when developing implementation interventions. Describing the process in steps ensured transparency and replicability of the method that could be used to develop similar implementation interventions for stroke treatments or guideline implementations across different conditions. The use of researchers incorporated well-informed judgment, acknowledged to be an important part of the process, especially for complex interventions [35]. The enabler data provided the researchers with valuable context-specific information to assist in the decision-making process. Reporting the BCT's using a standard taxonomy for by each $\mathrm{T}^{3}$ Trial behaviour has produced a well-described, reproducible and testable implementation intervention.

\section{Further research}

This study used a pragmatic approach to selecting BCTs that involved the $\mathrm{T}^{3}$ investigators who were familiar with the clinical protocols and clinical context. However, there is little evidence on who is best placed to make these judgments and what methods should be used. Michie et al. has developed the 'APEASE' criteria (A: Affordability; P: Practicability; E: Effectiveness/cost-effectiveness; A: Acceptability; S: Side-effects/Safety; E: Equality). However, it is unlikely that this information will be available for all the BCTs [7]. The $\mathrm{T}^{3}$ Trial is one of the few studies that have reported BCTs that were considered inappropriate for use (Table 3). Knowing more about the BCTs that are inappropriate for certain clinical contexts or even healthcare settings generally would be useful to make the overall pool of BCTs to choose from more relevant and straightforward. The Cane et al. matrix [20] is limited in suggesting BCTs for 
Table 4 Theory-informed implementation intervention: components by selected behavioural change techniques

\begin{tabular}{|c|c|}
\hline $\begin{array}{l}\text { Implementation } \\
\text { intervention component }\end{array}$ & $\begin{array}{l}\text { Selection of behavioural } \\
\text { change techniques }\end{array}$ \\
\hline \multirow{9}{*}{$\begin{array}{l}\text { Multidisciplinary barrier and } \\
\text { enabler workshops for ED, stroke } \\
\text { unit and endocrine clinicians }\end{array}$} & $\begin{array}{l}\text { Goal/target specified: } \\
\text { behaviour or outcome }\end{array}$ \\
\hline & $\begin{array}{l}\text { Social and environmental } \\
\text { consequences }\end{array}$ \\
\hline & $\begin{array}{l}\text { Restructuring the social } \\
\text { environment }\end{array}$ \\
\hline & $\begin{array}{l}\text { Environmental changes (e.g. } \\
\text { objects to facilitate behaviour) }\end{array}$ \\
\hline & Social support (practical) \\
\hline & Social support (emotional) \\
\hline & Planning, implementation \\
\hline & Action planning \\
\hline & Goal setting (behaviour) \\
\hline \multirow{11}{*}{$\begin{array}{l}\text { Interactive and didactic } \\
\text { education programme for } \\
\text { ED and stroke unit clinicians }\end{array}$} & Health consequences \\
\hline & Behavioural rehearsal/practice \\
\hline & $\begin{array}{l}\text { Social and environmental } \\
\text { consequences }\end{array}$ \\
\hline & Salience of consequences \\
\hline & Feedback on behaviour \\
\hline & Focus on past success \\
\hline & Social comparison \\
\hline & Reduce negative emotions \\
\hline & Anticipated regret \\
\hline & Coping skills \\
\hline & $\begin{array}{l}\text { Comparative imaging of } \\
\text { future outcomes }\end{array}$ \\
\hline \multirow[t]{7}{*}{ Use of local clinical opinion leaders } & $\begin{array}{l}\text { Verbal persuasion to boost } \\
\text { self-efficacy }\end{array}$ \\
\hline & Persuasive communication \\
\hline & Pros and cons \\
\hline & $\begin{array}{l}\text { Modelling/demonstration } \\
\text { of the behaviour }\end{array}$ \\
\hline & Anticipated regret \\
\hline & Social comparison \\
\hline & $\begin{array}{l}\text { Information about others' } \\
\text { approval }\end{array}$ \\
\hline \multirow[t]{2}{*}{ Reminders } & Prompts/cues \\
\hline & $\begin{array}{l}\text { Avoidance/changing exposure } \\
\text { to cues for the behaviour }\end{array}$ \\
\hline \multirow[t]{6}{*}{ Site support } & Self-monitoring \\
\hline & Self-reward \\
\hline & $\begin{array}{l}\text { Social support or } \\
\text { encouragement }\end{array}$ \\
\hline & Coping skills \\
\hline & Action planning \\
\hline & Goal setting \\
\hline
\end{tabular}

Technique may be classified to more than one implementation intervention component, e.g. action planning two of the TDF domains (social/professional role and identity; memory, attention and decision process). Therefore, further work should be conducted to assign more BCTs to these two domains. We acknowledge that research is currently underway which aims to link BCTs with theoretical mechanisms to better understand how interventions exert their effect and how to apply theory in implementation intervention development [36]. It is anticipated the approach used in this paper to develop and report an implementation intervention may contribute to the advancement of use of theory to guide intervention development. The TDF was a useful tool to map barriers to theoretical domains; however, as highlighted in this paper, inconsistencies between researchers can arise. Therefore, further work to clarify TDF domain definitions and to provide example barriers for each domain would complement this mapping process and minimise disagreements.

\section{Conclusion}

The TDF was successfully applied in all steps of developing an implementation intervention for the $\mathrm{T}^{3}$ Trial clinical protocols. The use of researcher opinion was valuable for the BCT selection process in terms of incorporating research evidence and well-informed judgment and incorporating the important practical issues of feasibility and acceptability. However, further recommendations are needed to advance understanding of who is best placed to inform implementation intervention development, and how best to incorporate this well-informed judgment. There is also a need to devise criteria for use in this BCT selection process. It is recommended that BCTs are classified by recognised implementation intervention components to facilitate generalisability and sharing across different conditions and settings.

\section{Additional files}

Additional file 1: Resource 1: barrier extracts and TDF definitions. (DOCX $23 \mathrm{~kb}$ )

Additional file 2: Resource 2: domains with corresponding $B C T$ and definitions. (DOCX 25 kb)

Additional file 3: Resource 3: technique definition and examples. (DOCX $71 \mathrm{~kb}$ )

Additional file 4: Resource 4: enabler data from the barrier and enabler workshops. (DOCX 26 kb)

Additional file 5: Resource 5: Cochrane Effective Practice and Organisation of Care [EPOC] reviews. (DOCX 25 kb)

Acknowledgements

Not applicable.

Funding

This Trial is funded by a National Health and Medical Research Council (NHMRC) Project Grant 1024812 (2012-2017). The following authors received research fellowship funding from the NHMRC: DC (co-funded with Heart 
Foundation: 1063761) and CL (Practitioner: 1043913). JG holds a Canada Research Chair in Health Knowledge Transfer and Uptake.

\section{Availability of data and materials}

Not applicable.

\section{Authors' contributions}

The following authors were responsible for obtaining funding or contributed to the research idea or study design: SM, CL, MF, JC, DC, EM, JG, RG, NWC, JW, LEC and NT. Data were collected by SM and SD. Workshop data were analysed and interpreted by LC, RP and NT. The researcher panel consisted of the following $\mathrm{T}^{3}$ Trial investigators and collaborators: SM, RG, DC, RP and Ms Enna Salama (ES). LC wrote the first draft of the paper with contributions from NT, RG, DC, EM, RP, SD, DOC and SM. All authors revised and approved the final manuscript.

\section{Ethics approval and consent to participate}

Approval from the Human Ethics Research Committee was initially obtained from Australian Catholic University (2012 16N), Sydney Local Health (Royal Prince Alfred Hospital Zone) (HREC/12/RPAH/32) (NSW Lead Ethics Committee). All participating hospitals have subsequently provided ethical governance clearances prior to data collection.

\section{Consent for publication}

Not applicable.

\section{Competing interests}

During the duration of the trial and following receipt of funding for the $\mathrm{T}^{3}$ Trial, SM was appointed to the Research Committee of the National Health \& Medical Research Council. DOC is an Associate Editor for Implementation Science. Other authors declare that there are no competing interests.

\section{Publisher's Note}

Springer Nature remains neutral with regard to jurisdictional claims in published maps and institutional affiliations.

\section{Author details}

${ }^{1}$ Nursing Research Institute, St Vincent's Health Australia (Sydney) and Australian Catholic University, Executive Suite, Level 5 deLacy Building, St Vincent's Hospital, 390 Victoria Street, Darlinghurst 2010, New South Wales, Australia. ${ }^{2}$ Cancer Research Division, Cancer Council NSW, 153 Dowling St, Woolloomooloo, NSW 2011, Australia. ${ }^{3}$ Sunshine Coast Hospital and Health Service/Sunshine Coast Clinical School, The University of Queensland, Nambour, QLD, Australia. ${ }^{4}$ Stroke and Ageing Research, School of Clinical Sciences at Monash Health, Monash University, Clayton, Victoria, Australia. ${ }^{5}$ Florey Institute of Neuroscience and Mental Health, University of Melbourne, Parkville, Victoria, Australia. ${ }^{6}$ School of Public Health and Preventive Medicine, Monash University, Level 1, 549 St Kilda Road, Melbourne, VIC, Australia. ${ }^{7}$ John Hunter Hospital, Newcastle, Australia. ${ }^{8}$ Centre for Translational Neuroscience and Mental Health, University of Newcastle/Hunter Medical Research Institute, Newcastle, Australia. ${ }^{9}$ Alfred Hospital, Melbourne, Victoria 3004, Australia. ${ }^{10}$ Department of Surgery, Central Clinical School, Monash University, Melbourne, Australia. ${ }^{11}$ National Trauma Research Institute, Melbourne, Australia. ${ }^{12}$ School of Nursing and Midwifery and Centre for Quality and Patient Safety Research - Eastern Health Partnership, Deakin University, Geelong, Victoria 3220, Australia. ${ }^{13}$ Clinical Epidemiology Program, Ottawa Health Research Institute, 1053 Carling Avenue, Administration Building, Room 2-017, Ottawa, Ontario K1Y 4E9, Canada. ${ }^{14}$ Department of Medicine, University of Ottawa, 451 Smyth Road, Ottawa, Ontario K1H 8M5, Canada. ${ }^{15}$ Department of Medicine, Monash University, Neurosciences Clinical Institute, Epworth hospital, Richmond, Victoria 3121, Australia. ${ }^{16}$ Centre for Diabetes and Endocrinology Research, Westmead Hospital and University of Sydney, Westmead, Sydney, NSW, Australia. ${ }^{17}$ School of Epidemiology, Public Health and Preventive Medicine (SEPHPM), University of Ottawa, 451 Smyth Road, Ottawa, Ontario K1H 8M5, Canada. ${ }^{18}$ Nulungu Research Institute, University of Notre Dame Australia, Broome, Western Australia, Australia.
Received: 19 December 2016 Accepted: 28 June 2017 Published online: 17 July 2017

\section{References}

1. National Stroke Foundation. Clinical Guideline for Stroke Management 2010.

2. Mosley I, Morphet J, Innes K, Braitberg G. Triage assessments and the activation of rapid care protocols for acute stroke patients. Australas Emerg Nurs J. 2013;16(1):4-9.

3. Middleton S, Levi C, Dale S, Cheung NW, Mclnnes E, Considine J, et al. Triage, treatment and transfer of patients with stroke in emergency department trial (the T3 Trial): a cluster randomised trial protocol. Implement Sci. 2016;11(1):139.

4. Craig P, Dieppe P, Macintyre S, Michie S, Nazareth I, Petticrew M. Developing and evaluating complex interventions: the new medical research council guidance. BMJ. 2008;337:a1655.

5. Michie S, Johnston M, Francis J, Hardeman W, Eccles M. From theory to intervention: mapping theoretically derived behavioural determinants to behaviour change techniques. Appl Psychol. 2008;57:660-80.

6. French S, Green S, O'Connor D, McKenzie J, Francis J, Michie S, et al. Developing theory-informed behaviour change interventions to implement evidence into practice: a systematic approach using the Theoretical Domains Framework. Implement Sci. 2012;7(1):38.

7. Michie S, van Stralen MM, West R. The behaviour change wheel: a new method for characterising and designing behaviour change interventions. Implement Sci. 2011;6(1):1-12. doi:10.1186/1748-5908-6-42.

8. Sinnott C, Mercer SW, Payne RA, Duerden M, Bradley CP, Byrne M. Improving medication management in multimorbidity: development of the MultimorbiditY COllaborative Medication Review And DEcision Making (MY COMRADE) intervention using the Behaviour Change Wheel. Implement Sci. 2015;10:132.

9. Tavender EJ, Bosch M, Gruen RL, Green SE, Michie S, Brennan SE, et al. Developing a targeted, theory-informed implementation intervention using two theoretical frameworks to address health professional and organisational factors: a case study to improve the management of mild traumatic brain injury in the emergency department. Implement Sci. 2015;10:74.

10. Taylor N, Lawton R, Slater B, Foy R. The demonstration of a theory-based approach to the design of localized patient safety interventions. Implement Sci. 2013;8:123.

11. Eccles MP, Johnston M, Hrisos S, Francis J, Grimshaw J, Steen N, et al. Translating clinicians' beliefs into implementation interventions (TRACII): a protocol for an intervention modeling experiment to change clinicians' intentions to implement evidence-based practice. Implement Sci. 2007;2:27.

12. Hoffmann TC, Glasziou PP, Boutron I, Milne R, Perera R, Moher D, et al. Better reporting of interventions: template for intervention description and replication (TIDieR) checklist and guide. BMJ. 2014;348:g1687.

13. Michie S, Johnston M, Abraham C, Lawton R, Parker D, Walker A, et al. Making psychological theory useful for implementing evidence based practice: a consensus approach. Qual Saf Health Care. 2005;14(1):26-33.

14. Cane J, O'Connor D, Michie S. Validation of the theoretical domains framework for use in behaviour change and implementation research. Implement Sci. 2012;7(1):37.

15. McKenzie JE, French SD, O'Connor DA, Grimshaw JM, Mortimer D, Michie S, et al. IMPLEmenting a clinical practice guideline for acute low back pain evidence-based manageMENT in general practice (IMPLEMENT): cluster randomised controlled trial study protocol. Implement Sci. 2008;3:11.

16. Steinmo S, Fuller C, Stone SP, Michie S. Characterising an implementation intervention in terms of behaviour change techniques and theory: the 'Sepsis Six' clinical care bundle. Implement Sci. 2015;10:111.

17. Steinmo SH, Michie S, Fuller C, Stanley S, Stapleton C, Stone SP. Bridging the gap between pragmatic intervention design and theory: using behavioural science tools to modify an existing quality improvement programme to implement "Sepsis Six". Implement Sci. 2016;11:14.

18. Patey AM, Islam R, Francis JJ, Bryson GL, Grimshaw JM, Canada PPT. Anesthesiologists' and surgeons' perceptions about routine pre-operative testing in low-risk patients: application of the Theoretical Domains Framework (TDF) to identify factors that influence physicians' decisions to order pre-operative tests. Implement Sci. 2012;7:52.

19. Connell LA, McMahon NE, Watkins CL, Eng JJ. Therapists' use of the Graded Repetitive Arm Supplementary Program (GRASP) intervention: a practice implementation survey study. Phys Ther. 2014;94(5):632-43. 
20. Cane J, Richardson M, Johnston M, Ladha R, Michie S. From lists of behaviour change techniques (BCTs) to structured hierarchies: comparison of two methods of developing a hierarchy of BCTs. Brit J of Health Psych. 2015;20(1):130-50.

21. Davidoff F, Dixon-Woods M, Leviton L, Michie S. Demystifying theory and its use in improvement. BMJ Qual Saf. 2015;24(3):228-38.

22. McCullough AR, Ryan C, O'Neill B, Bradley JM, Elborn JS, Hughes CM. Defining the content and delivery of an intervention to Change AdhereNce to treatment in BonchiEctasis (CAN-BE): a qualitative approach incorporating the Theoretical Domains Framework, behavioural change techniques and stakeholder expert panels. BMC Health Serv Res. 2015;15:342.

23. Middleton S, McElduff P, Ward J, Grimshaw JM, Dale S, D'Este C, et al. Implementation of evidence-based treatment protocols to manage fever, hyperglycaemia, and swallowing dysfunction in acute stroke (QASC): a cluster randomised controlled trial. Lancet. 2011:378(9804):1699-706.

24. Michie S, Richardson M, Johnston M, Abraham C, Francis J, Hardeman W, et al. The behavior change technique taxonomy (v1) of 93 hierarchically clustered techniques: building an international consensus for the reporting of behavior change interventions. Ann Behav Med. 2013;46(1):81-95.

25. Flodgren G, Parmelli E, Doumit G, Gattellari M, O'Brien MA, Grimshaw J et al. Local opinion leaders: effects on professional practice and health care outcomes. Cochrane Database Syst Rev. 2011;(8):CD000125.

26. Forsetlund L, Bjørndal A, Rashidian A, Jamtvedt G, O'Brien MA, Wolf FM et al. Continuing education meetings and workshops: effects on professional practice and health care outcomes. Cochrane Database Syst Rev. 2009;(2): CD003030.

27. Giguère A, Légaré F, Grimshaw J, Turcotte $S$, Fiander $M$, Grudniewicz A et al. Printed educational materials: effects on professional practice and healthcare outcomes. Cochrane Database Syst Rev. 2012;(10):CD004398.

28. Ivers N, Jamtvedt G, Flottorp S, Young JM, Odgaard-Jensen J, French SD et al. Audit and feedback: effects on professional practice and healthcare outcomes. Cochrane Database Syst Rev. 2012;(6):CD000259.

29. O'Brien MA, Rogers S, Jamtvedt G, Oxman AD, Odgaard-Jensen J, Kristoffersen DT et al. Educational outreach visits: effects on professional practice and health care outcomes. Cochrane Database Syst Rev. 2007;(4): CD000409.

30. Shojania KG, Jennings A, Mayhew A, Ramsay CR, Eccles MP, Grimshaw J. The effects of on-screen, point of care computer reminders on processes and outcomes of care. Cochrane Database Syst Rev. 2009;(3):CD001096.

31. Cosby JL. Improving Patient Care: The Implementation of Change in Clinical Practice. Qual Saf Health Care. 2006;15(6):447.

32. Forsetlund L, Bjorndal A, Rashidian A, Jamtvedt G, O'Brien MA, Wolf F, et al. Continuing education meetings and workshops: effects on professional practice and health care outcomes. Cochrane Database Syst Rev. 2009;2: CD003030.

33. O'Brien MA, Rogers $\mathrm{S}$, Jamtvedt G, Oxman AD, Odgaard-Jensen J, Kristoffersen DT, et al. Educational outreach visits: effects on professional practice and health care outcomes. Cochrane Database Syst Rev. 2007:4: CD000409.

34. Grimshaw JM, Thomas RE, MacLennan G, Fraser C, Ramsay CR, Vale L, et al. Effectiveness and efficiency of guideline dissemination and implementation strategies. Health Technol Asses. 2004;8(6):iii-iv. 1-72.

35. Dixon-Woods M. The problem of context in quality improvement. In: Perspectives on context. Health Foundation. 2014.

36. University of College London. Centre for Behaviour Change. http://www.ucl ac.uk/behaviour-change. Accessed 25 Nov 2016.

\section{Submit your next manuscript to BioMed Central and we will help you at every step:}

- We accept pre-submission inquiries

- Our selector tool helps you to find the most relevant journal

- We provide round the clock customer support

- Convenient online submission

- Thorough peer review

- Inclusion in PubMed and all major indexing services

- Maximum visibility for your research

Submit your manuscript at www.biomedcentral.com/submit
Biomed Central 